\title{
Therapeutic embolisation of the external carotid arterial tree
}

\author{
BRIA K K NDALLAND IVAN MOSELEY \\ From the Lysholm Radiological Department, National Hospital for Nervous Diseases, Queen Square, \\ London
}

SUMMARY Intra-arterial embolisation is a valuable adjunct to the treatment of many vascular lesions, including neoplasms such as glomus tumours or juvenile angiofibromas, and arteriovenous malformations. Its place in the management of the individual patient should be established before any surgical procedure is carried out, as this may prejudice the eventual result. The indications for the procedure, the technique, and possible complications are discussed in this paper, and it is emphasised that the latter are best avoided by the use of a scrupulous technique and adequate technical facilities.

Therapeutic intra-arterial embolisation of vascular lesions is not a new technique; it was suggested in 1930 by Brooks, at a time when carotid angiography itself had been practised for only three years. Since then it has been used extensively as an adjunct to surgery or as definitive therapy, and the purpose of the present article is to describe some of its applications and advantages in the management of vascular lesions of the neck and head.

The aim of intravascular embolisation is to occlude the blood vessels of a tumour or other vascular lesion which is either inaccessible to surgery, or in which surgery could involve considerable haemorrhage, within the lesion itself or at a critical level. Thus, in the case of a vascular malformation or tumour in the external carotid territory, ligation or occlusion of feeding vessels at some distance from the shunt or tumour bed has repeatedly been shown to result in the rapid development of collateral supply; the placement of emboli within the pathological vessels themselves precludes this.

In the external carotid territory embolisation may be useful in the management of angiomas: of the skin, tongue, bone, or dura mater; of arteriovenous fistulae, eg caroticocavernous; and of tumours: glomus tumours, angiofibromas, meningiomas, or malignant tumours. Similar lesions

\footnotetext{
${ }^{1}$ Address for reprint requests: Dr I. F. Moseley, The National Hospital, Queen Square, London WCiN 3BG.

Accepted 16 April 1977
}

can, of course, be treated in other parts of the body.

The contraindications to embolisation include allergy to angiographic contrast medium (but see case 6), and atheroma of the carotid bifurcation, which precludes selective catheterisation. There is little indication for the treatment of lesions which present no difficulty at surgery and are unlikely to produce poor cosmetic results.

Brooks (1930) recommended muscle as the embolic material, while later workers have used radio-opaque metallic, plastic, or silicone spheres (Luessenhop, 1969) or Gelfoam fragments (Ishimori et al., 1967). More recently, rapidly solidifying liquids have been prepared for embolisation. We have consistently used fragments of Gelfoam, which is non-antigenic. These have the advantages of being relatively cheap and simple to handle and prepare; their size can be finely adjusted to the individual case. Injection of even large fragments is easy and does not require a large catheter. Although they appear to provide a satisfactory thrombosis, we feel that a single fragment misplaced in a vessel which it is not intended to embolise may be less harmful than a rigid metal or plastic sphere. The use of rapidly solidifying liquids, although very effective, may produce a more complete occlusion than is desirable of some of the tiny normal vessels arising from branches of the external carotid artery to supply the cranial nerves.

Preparation of the patient is important. If the external carotid artery is to be embolised, it is 
essential that the patient should understand that there is a risk of permanent neurological deficit, very minor though this may be; full informed consent must, therefore, be given. The procedure may be carried out under local or general anaesthesia: the former has the advantage that the neurological state of the patient may be monitored continuously, the latter that pain on injection of the external carotid artery branches, which may cause the patient to move and impair the diagnostic quality of the radiographs, is avoided. Embolisation of a complex lesion may also be a lengthy procedure. As a rule we use general anaesthesia.

The catheter through which embolisation is to be carried out may be introduced by direct external or common carotid puncture, or by retrograde Seldinger catheterisation of the axillary or femoral arteries. We use the last approach which has three main advantages: it is the one through which manipulation of the catheter is easiest, the operator's hands are kept far from the radiation field, and all the head and neck vessels may be injected after a single arterial puncture.

Adequate fluoroscopic and radiographic apparatus is also very important. We use a caesium iodide image intensifier which can be used in the lateral position for fluoroscopy, and the angiographic series are carried out with $2 \times$ or $3 \times$ magnification, using a $0.2 \mathrm{~mm}$ focal spot tube. Any compromise on apparatus may prejudice the efficacy and safety of the procedure.

In adults we use the standard flexible, thinwalled $5 \mathrm{~F}$ gauge catheters, while in young children, smaller tubing may be used. The first stage of the procedure is filming of the carotid bifurcation to exclude ulcerating or stenosing atheroma, followed by the documentation of the vascular supply of the lesion to be embolised, and it is our practice to inject first those vessels of which embolisation is unlikely to be possible-that is, the internal carotid and vertebral arteries. If most of the blood supply to the lesion arises from these vessels, or if there are widely patent anastomoses between them and the external carotid branches, embolisation is not feasible (Figs. 1, 2).

Next, one of the external carotid artery branches supplying the lesion is catheterised; lateral fluoroscopy is particularly useful during this superselective catheterisation. An angiographic series is then performed and this is inspected before embolisation to determine: (i) the degree and nature of pathological vascularitythat is, the presence or absence of significant arteriovenous shunting: if there is no pathological vascular bed, emboli will pass directly through into the venous circulation; (ii) the size of the vessels feeding the lesion and the volume of flow within them: this will determine the size of the emboli to be injected and the speed of the injection; (iii) the presence of anastomoses with the internal carotid or vertebrobasilar trees, which will contraindicate embolisation; and (iv) the superselectivity of the catheterisation, and lack of reflux into the internal carotid or vertebral circulations.

If the angiographic appearances are satisfactory for embolisation, small fragments of Gelfoam are cut with fine scissors, the size depending on that of the vessels to be occluded, and suspended in non-heparinised saline. The syringes which are used for injecting the emboli are distinctively marked, and are used for this purpose only. Just before embolisation, and repeatedly during the procedure, the catheter is inspected fluoroscopically to ensure that it has not moved. Ten to 20 emboli are then injected, followed by a small quantity of contrast material to check fluoroscopically that the vessel is still patent and that reflux is not occurring.

This procedure is repeated, using progressively larger emboli, until it is clear at fluoroscopy that the flow in the catheterised vessel has slowed significantly. Another angiogram is then obtained. If this demonstrates almost complete occlusion of the pathological vessels, no further emboli are injected; it is not the aim to achieve complete blockage of the feeding vessel at this stage as this may be hazardous. If there is no flow, marked spasm, a proximal block, or obvious reflux, embolisation of that vessel is also terminated, for the time being at least.

Successful embolisation depends in part on preferential flow towards vascular lesions; when this is abolished, there is no force directing the emboli to their desired site. The same procedure is carried out until all the feeding vessels have been dealt with, although with very extensive lesions it may be necessary to carry out embolisation in several stages.

Some workers have suggested the injection of a sclerosant or thrombogenic agent at the end of the embolisation of each vessel (Djindjian, 1977) but we have not found this necessary.

When embolisation appears complete, a flush injection is made into the external carotid artery. This will demonstrate any remaining vascular lesions, and in many cases, by reflux, confirm that the internal carotid artery remains normally patent. 


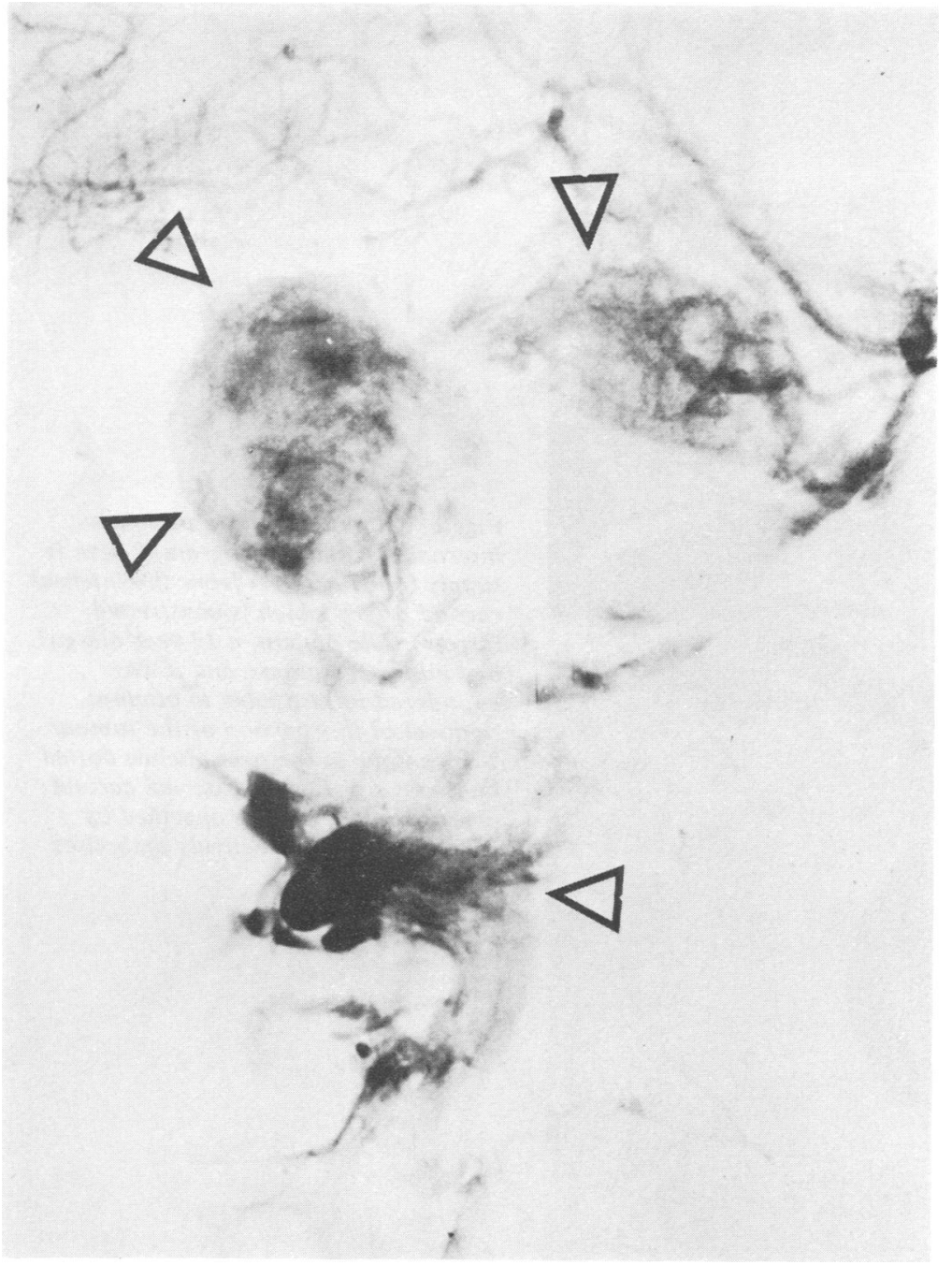

Fig. 1 Glomus jugulare tumour-vertebral angiogram. (All illustrations are lateral projections, subtracted, the right margin being anterior.) There is massive supply to the tumour (open arrowheads). External carotid supply was negligible, and the lesion was not treated by embolisation.

The following eight case reports illustrate the indications for the procedure and the modifications to the basic technique which may be necessary.

\section{Case 1 Facial angioma}

PL, a 30 year old moneybroker, complained of intermittent bleeding from a lump affecting the left upper lip and alveolus. There was also recurrent gingivitis which had caused loosening of his front teeth and it was now considered necessary to remove these. Because of the danger of torrential haemorrhage he was referred for embolisation in order to reduce the blood supply to the superior alveolus. Examination confirmed an extensive angiomatous malformation of the left upper lip and alveolus.

On 14 November 1975 superselective angiography showed that the malformation was supplied by the superior alveolar, major palatine and sphenopalatine branches of the maxillary arteries, and by branches of both facial arteries. There was no supply from other vessels. Gelfoam emboli were injected into the branches supplying the lesion, and repeat angiography at the termination of the procedure showed that there was only a very minor residuum (Fig. 3).

Over the 24 hours after embolisation there was 


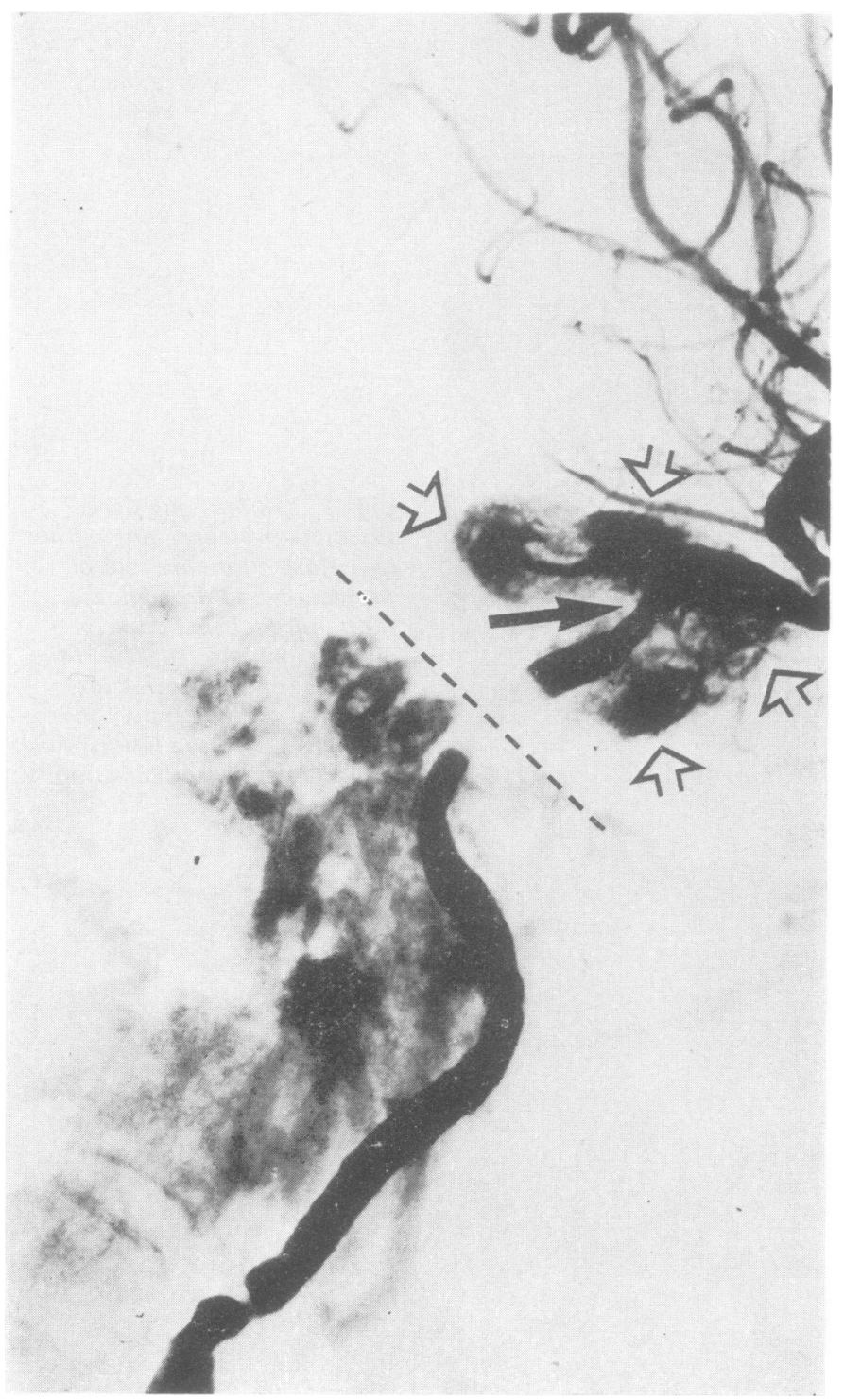

Fig. 2 Glomus jugulare tumourinternal carotid arteriogram. There is supply (open arrows) from the internal carotid artery which is constricted (arrow). The patient, a 19 year old girl, had minor symptoms, and it was considered unjustifiable to attempt removal of this portion of the tumour. The tumour in the neck (below dotted line) was supplied by external carotid artery branches and is opacified by reflux. This was effectively embolised.

progressive and considerable reduction in the size of the malformation and there were no adverse effects. Four days later the patient complained of a feeling of stiffness in the region of the angioma, but this soon abated. The patient's teeth were removed without undue haemorrhage.

\section{Case 2 Facial angioma}

AM, a 22 year old housewife, complained of a small fluctuant swelling of the left eyelid and the adjacent malar region. At angiography, carried out before cosmetic surgery to determine the blood supply, this was seen to be an angioma, largely supplied by the superficial temporal artery, with a small contribution from the supraorbital branch of the ophthalmic artery.

The superficial temporal artery was then embolised (Fig. 4). The object of embolisation in this case was merely to facilitate surgery, but it was so effective that no operation was required. 


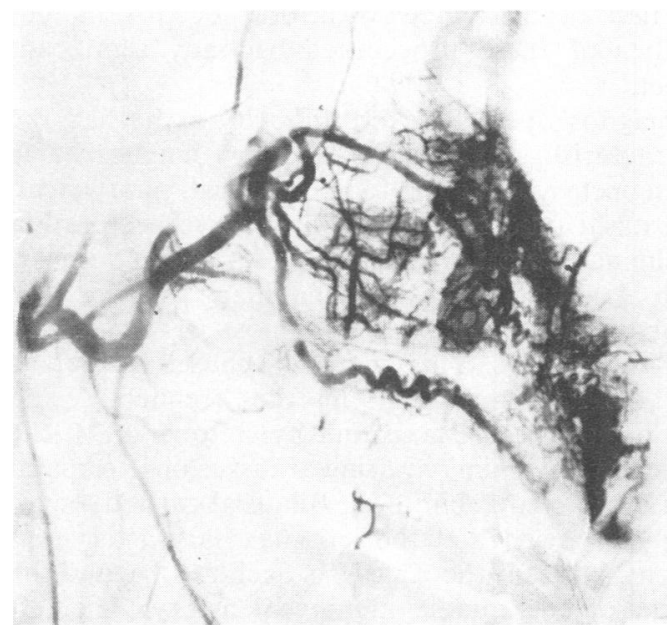

(a)

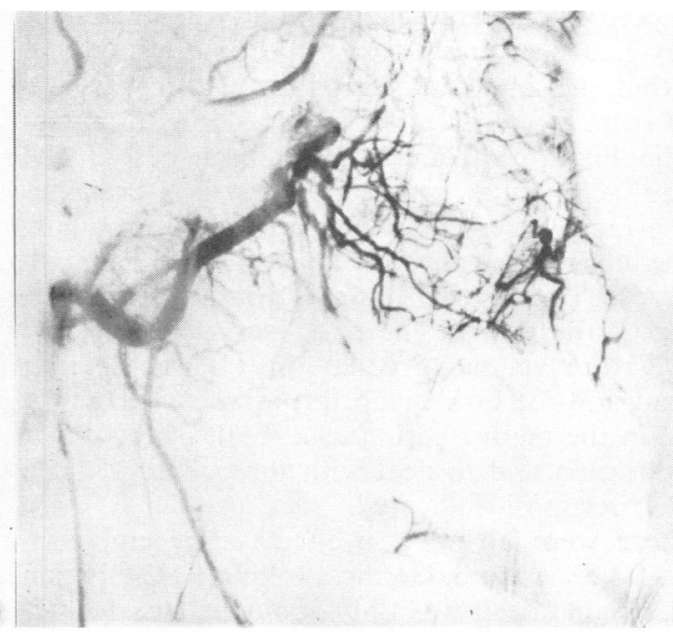

(b)

Fig. 3 Case 1 Facial angioma-selective injection of the maxillary artery. (a) Before embolisation-massive angioma of face and upper lip, supplied by hypertrophied infraorbital and descending palatine vessels; (b) after embolisation-many normal vessels are preserved.

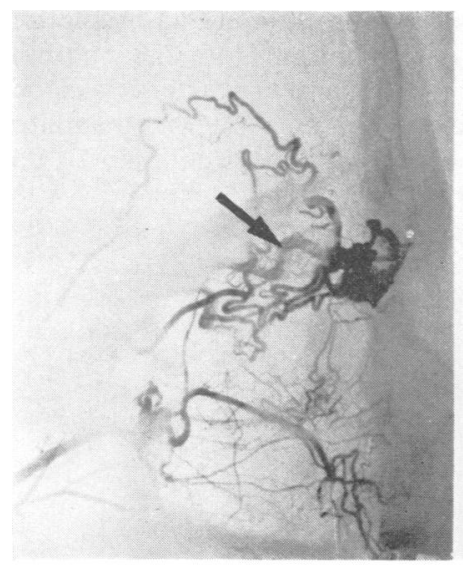

(a)

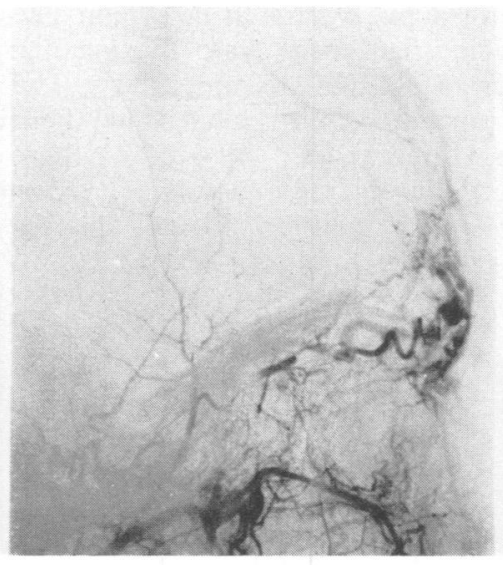

(b)

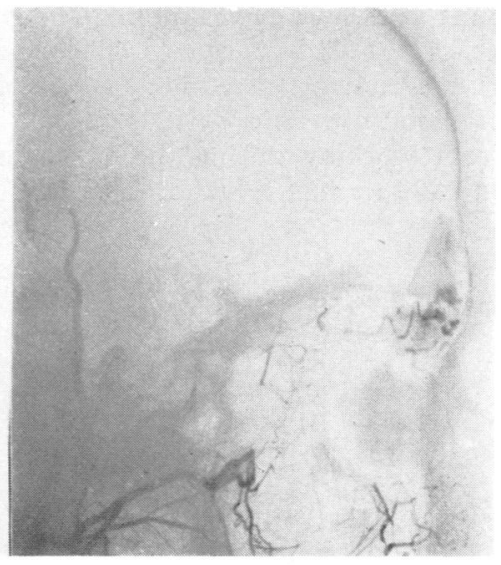

(c)

Fig. 4 Case 3 Facial angioma-injection of maxillary and superficial temporal arteries. (a) Before embolisation-the angioma is largely fed by superficial temporal artery; main venous drainage is towards superior ophthalmic vein (arrows); (b) during embolisation-partial obliteration of lesion; (c) final seriesonly a small portion of the angioma remains, fed by tiny collateral vessels from maxillary artery.

\section{Case 3 Angioma of right parotid region}

$\mathrm{RN}$, a 30 year old housewife, complained of a swelling in the right cheek and tonsillar region for two and a half months. Examination had suggested that the swelling was within the right parotid gland, and exploration and partial excision showed that the lesion was an angiomatous malformation. An angiogram had shown that residual malformation was supplied by branches of the external carotid artery and the patient was referred to us for embolisation.

Transfemoral superselective catheterisation of the branches of the right external carotid artery 
was performed. This showed that the maxillary artery had been ligated within the parotid gland, but that the angiomatous malformation was supplied from branches of the vessel arising proximal to the ligature, from tonsillar branches of the linguo-facial trunk and from masseteric branches of the facial artery. Virtually all the direct supply to the angioma was occluded with Gelfoam emboli (Fig. 5). There was, however, some retrograde filling of the part of the maxillary artery beyond the ligature via facial collaterals. It was decided to wait and see how much thrombosis would take place in the malformation as a result of the direct embolisation and to deal with the collaterals at a further session if indicated.

There were no adverse effects after embolisation. Three weeks later the swelling in the region of the right tonsil was only about half its former size and there was further reduction over the following months.

Case 4 Epistaxis from hereditary haemorrhagic telangiectasia

JB, a 54 year old male caretaker, was referred to King's College Hospital in 1974 with a five year history of daily epistaxes. The bleeding had been severe enough to require transfusion of 500 pints of blood over this period and had prevented him from working during the preceding three years. In 1972 he had a bilateral septal dermoplasty and he had been treated with oral oestrogens but neither of these procedures had any significant effect.

Diagnosis presented no difficulty as he had the characteristic lesions of hereditary haemorrhagic telangiectasia over the face, lips, oral, pharyngeal, and nasal mucosa. There was a large perforation of the nasal septum as a result of previous surgery.

In January 1974 both maxillary arteries and their terminal branches in the nose were occluded with silver clips. This operation caused a temporary marked reduction in the frequency and severity of the epistaxis, but by October 1974, the patient was again requiring transfusions to maintain his haemoglobin level. Bilateral carotid angiography showed that the left maxillary artery was patent, but that the right was occluded beyond the origin of the middle meningeal artery. The left ethmoidal, facial, lingual, and external carotid arteries were ligated. There was again marked but temporary reduction in the frequency and severity of the epistaxis, but they gradually returned to their previous severity and he was requiring transfusion by April 1976. Repeat angiography showed that the left external carotid artery was occluded at its origin. There was some collateral circulation into the maxillary distribution from the ophthalmic artery. The right maxillary artery was occluded in the region of the pterygo-maxillary fissure and there was collateral circulation to the nasal cavity from the right facial artery.

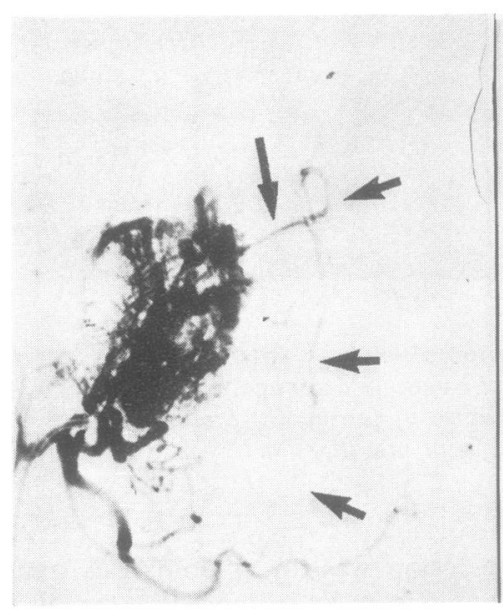

(a)

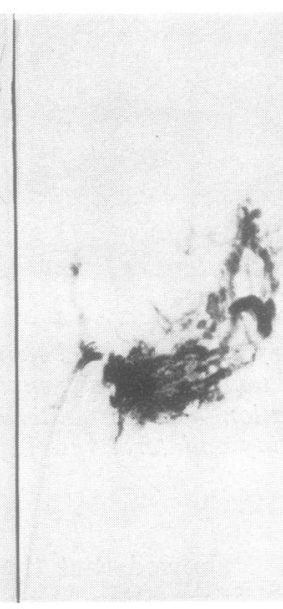

(b)

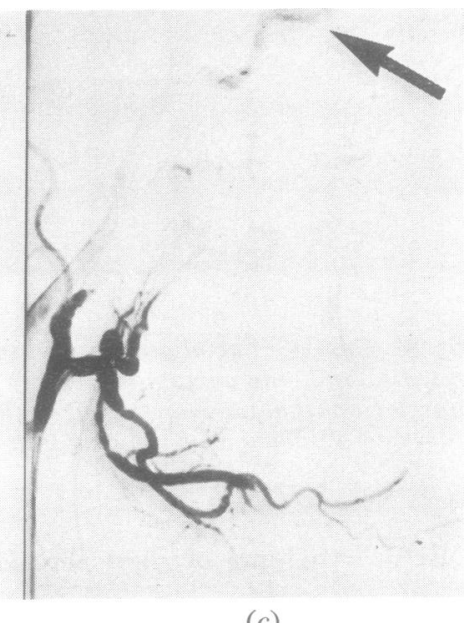

(c)

Fig. 5 Case 3 Parotid angioma-injection of linguofacial trunk. (a) Before embolisation-the angioma is supplied from facial artery. The proximally-ligated maxillary artery (long arrow) fills via collaterals (arrows); (b) during embolisation-the mass of the angioma is reduced; (c) final series-the angioma is almost completely obliterated. Flush injection confirms patency of internal carotid artery (arrow). 
On 4 June 1976 magnification angiography of the right maxillary artery showed telangiectases supplied by its remaining branches. The telangiectases and finally the supplying vessels were occluded with Gelfoam emboli. A right facial arteriogram showed that the vessel supplied collateral circulation into some nasal telangiectases. These were similarly embolised, but one small collateral branch was left patent because forward flow in the main trunk of the vessel had become sufficiently slow to cause a dangerous degree of reflux towards the internal carotid origin. No telangiectases were supplied by the ascending pharyngeal artery, and although the lingual artery did supply some on the tongue these were not causing haemorrhage and were not embolised.

There was some pain and tenderness over the anterior branch of the right superficial temporal artery which subsided over the next few days, but otherwise there were no symptoms due to the procedure. There was immediate cessation of the epistaxes but, unfortunately, these recurred and the patient died two months later. It should be noted that only a small proportion of the patient's lesions were amenable to embolisation because the direct vascular supply to other lesions had been ligated proximally and they were being supplied by collateral circulation through branches of the right opnthalmic artery.

\section{Case 5 Dural angiomatous malformation}

JP, a 21 year old housewife, complained of proptosis of the right eye for three years which had begun during pregnancy, but was non-progressive. There was a past history of head injury at the age of 14 years, which had been followed by a machinery murmur in the head. This had lasted for one year but then disappeared. Examination revealed marked proptosis with venous congestion of the right eyelid and a bruit over the right eye. External ocular movements were full. Visual acuity was $\mathrm{J} 1$ in both eyes and there was no papilloedema.

Skull radiographs showed enlargement of the right superior ophthalmic fissure and an EMI scan showed a dilated superior ophthalmic vein. Carotid angiography showed a fistula between the medial branch of the right middle meningeal artery and a vein draining into the right cavernous sinus. There was no supply from the internal carotid artery and none was evident from any other vessel. Superselective angiography confirmed that the fistula filled from this single vessel and showed marked early venous drainage into the cavernous sinus and superior ophthalmic veins.
Gelfoam emboli were injected into the right middle meningeal artery. These caused marked reduction in flow through the fistula and there was no early venous drainage at the termination of the procedure.

After embolisation the bruit disappeared and the proptosis was less marked. The patient was seen in the outpatient department one year later; she remains well, with no bruit and only minimal residual proptosis.

\section{Case 6 Post-traumatic left carotid cavernous sinus} fistulae

LE, a 23 year old housewife, complained of pulsating exophthalmos of the left eye which had been increasing for 10 days. In 1970 she suffered a head injury when she was knocked down by a car and this had resulted in immediate left proptosis and diplopia. The left internal carotid artery was ligated in the neck and the orbit virtually returned to normal. Ten days before admission she had a sudden left frontal headache associated with an intermittent subjective bruit behind the left eye.

Examination revealed bruits over both orbits. There was non-pulsatile left proptosis with engorgement of veins around the eye and over the forehead. Visual acuity and visual fields were normal, but fundoscopy showed venous engorgement and pulsation with early papilloedema on the left and venous engorgement on the right.

On 20 May 1976 bilateral carotid angiography was performed. After injection of contrast medium there was immediate marked hypotension associated with flushing and urticaria. Only a limited study was, therefore, performed and this revealed occlusion of the left internal carotid artery in the neck, with cross circulation from the right internal carotid artery and retrograde filling into the left cavernous sinus. There was only faint filling of the left middle cerebral artery and its branches.

After the angiogram the patient was neurologically normal, but over the next 24 hours there were episodes of dysphasia and right sided weakness. By 48 hours after the procedure the patient had returned to her preangiographic state.

On 25 May 1976 the left terminal carotid artery was occluded by clipping. The brain was noted to be swollen with venous engorgement, especially affecting the Sylvian veins. There was a left posterior communicating aneurysm which was clipped. Clipping of the terminal carotid artery caused a fall in the Sylvian venous pressure. A vein connecting the Sylvian vein to the cavernous sinus was clipped 
Fig. 6 Case 6 Post-traumatic caroticocavernous fistula-selective injection of the maxillary artery. There is filling of the dilated superior ophthalmic vein via cavernous sinus (open arrow). Catheter position is not suitable for embolisation, since middle meningeal artery also supplies ophthalmic artery (arrows). Catheter tip was advanced beyond the origin of this vessel before embolisation.

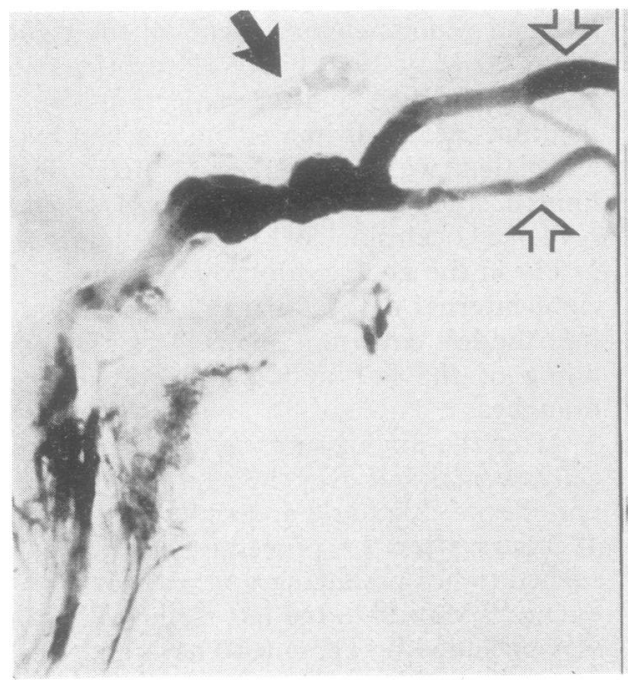

(a)

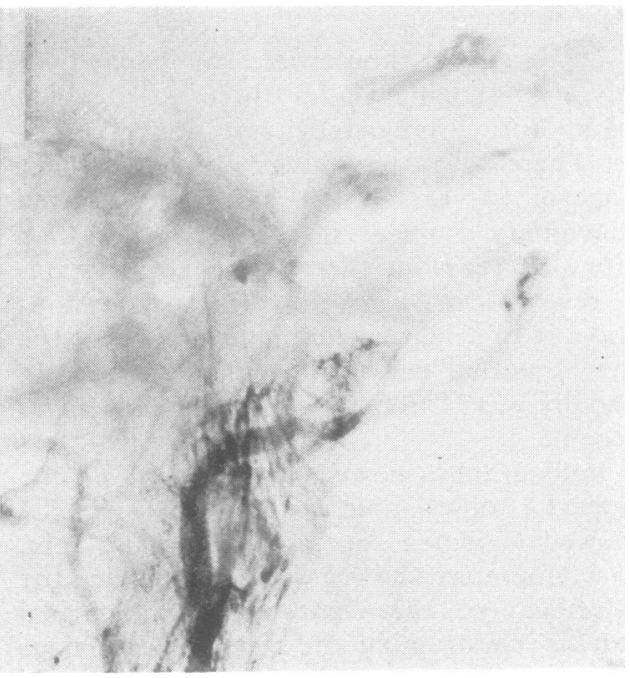

(b)

Fig. 7 Case 6 (a) Injection of ascending pharyngeal artery-there is dense opacification of venous sinuses and superior and inferior ophthalmic veins (open arrows). Note clips on internal carotid artery (arrow); (b) after embolisation-the shunt has been almost completely obliterated. 


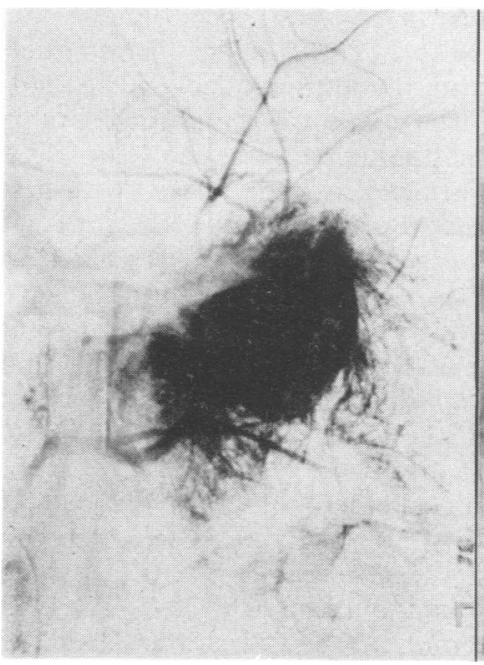

(a)

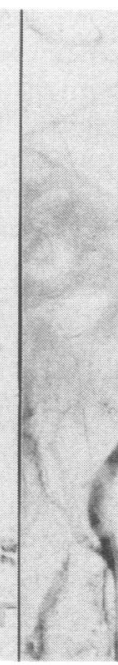

$\sin$

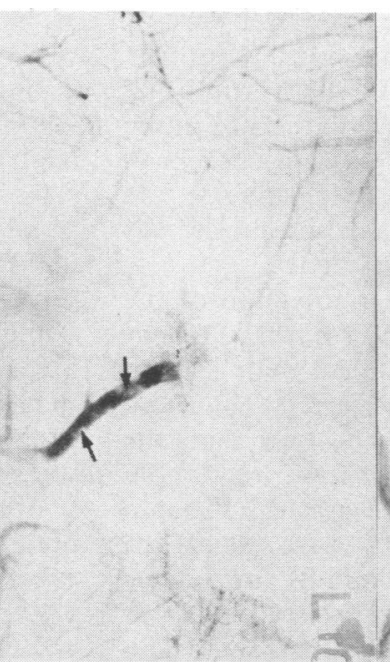

(b) (c)

Fig. 8 Case 7 Juvenile angiofibroma. Injection of the maxillary artery. (a) Before embolisation-part of tumour is shown; (b) during embolisation-a small amount of pathological circulation persists and emboli may be seen within artery (arrows); (c) final injection of maxillary artery-the vessel is occluded just beyond catheter tip (arrow).

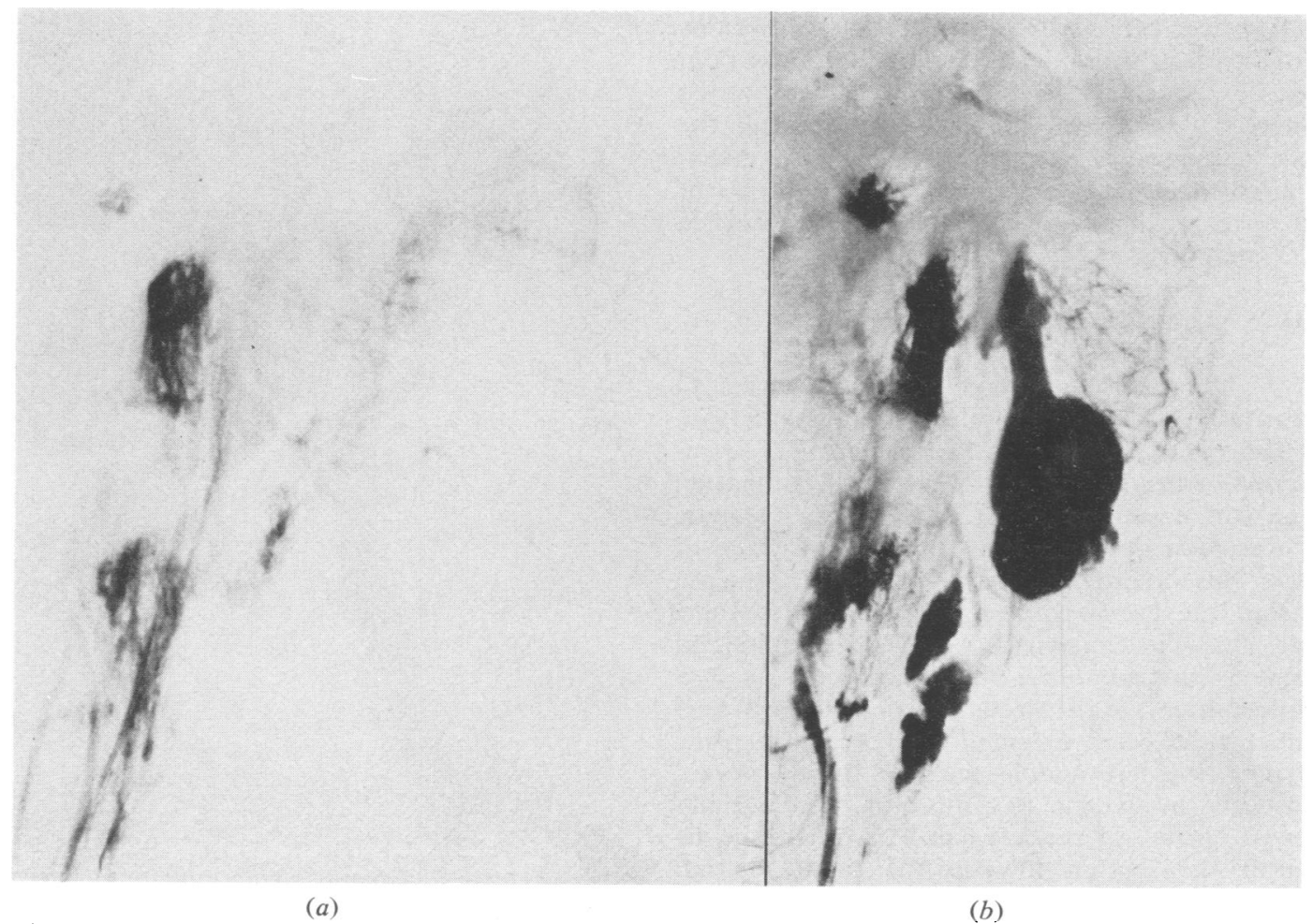

Fig. 9 Case 7 Injection of ascending pharyngeal artery. (a) A small amount of pathological circulation is seen; (b) after initial embolisation, test in jection has resulted in extravasation: amorphous collections of contrast medium are seen. 
and this raised pressure in the proximal part of the vein, with dilatation of the veins anteriorly in the temporal fossa, one of which ruptured. These vessels were occluded by diathermy along the edge of the sinus and this caused reduction in the Sylvian venous pressure. After surgery, the orbital bruit disappeared and the proptosis was much less. There was no cerebral deficit.

One month later superselective injection of the branches of the right external carotid artery showed that the fistula was supplied mainly from the right ascending pharyngeal artery, but also branches of the maxillary artery other than the middle meningeal artery which supplied the ophthalmic artery (Fig. 6). Emboli were injected into the ascending pharyngeal artery (Fig. 7) and the maxillary artery beyond the middle meningeal origin; towards the end of the procedure it was necessary to use large Gelfoam fragments. This resulted in a marked reduction in flow through the fistula. The procedure was ended when considerable reflux into muscular collaterals of the external carotid artery was shown and there was quite prominent filling of the vertebrobasilar circulation from these.

After the embolisation, the proptosis became gradually less prominent. The patient was considerably worried by painful trismus which gradually abated over the three weeks after the procedure. There was no other adverse effect. When seen at six monthly follow-up the patient had no complaints and the left eye had returned to normal.

\section{Case 7 Angiofibroma of the nasopharynx}

CS, a 12 year old schoolboy, was referred to Great Ormond Street Hospital in September 1976. He had been seen first in September 1975, complaining of headaches, at which time examination by an ear, nose, and throat surgeon was negative. He was seen again in May 1976 complaining of severe nose bleeds. There was necrotic mucosa on the free border of the left middle turbinate bone; this was cauterised. Complete examination failed to show any other abnormality.

Intermittent nose bleeds continued until September 1976 when examination revealed a white polyp in the left middle meatus. Biopsy proved this to be a juvenile angiofibroma. Radiographs showed opacity of the left nasal space with deviation of the septum towards the right; the left antrum was opaque.

Transfemoral selective internal carotid angiography and supraselective angiography of the maxillary, facial and ascending pharyngeal arteries of both sides were performed. The tumour was very vascular, with a pathological circulation derived mainly from the left maxillary and ascending pharyngeal arteries, but with a considerable contribution from the same vessels on the right side. These four arteries were embolised with first small and then progressively larger Gelfoam fragments, so as to occlude the tumour vessels themselves and then the supplying branches (Fig. 8). Some extravasation occurred from the right ascending pharyngeal artery during a test injection monitoring the embolisation (Fig. 9). This was not progressive and the procedure was otherwise uneventful.

For a few days after embolisation the patient complained of sore throat, but there were no other symptoms. Eleven days later a transpalatal excision of the angiofibroma was performed. It was attached to the roof and left lateral wall of the nasal cavity $10 \mathrm{~mm}$ anterior to the pterygoid

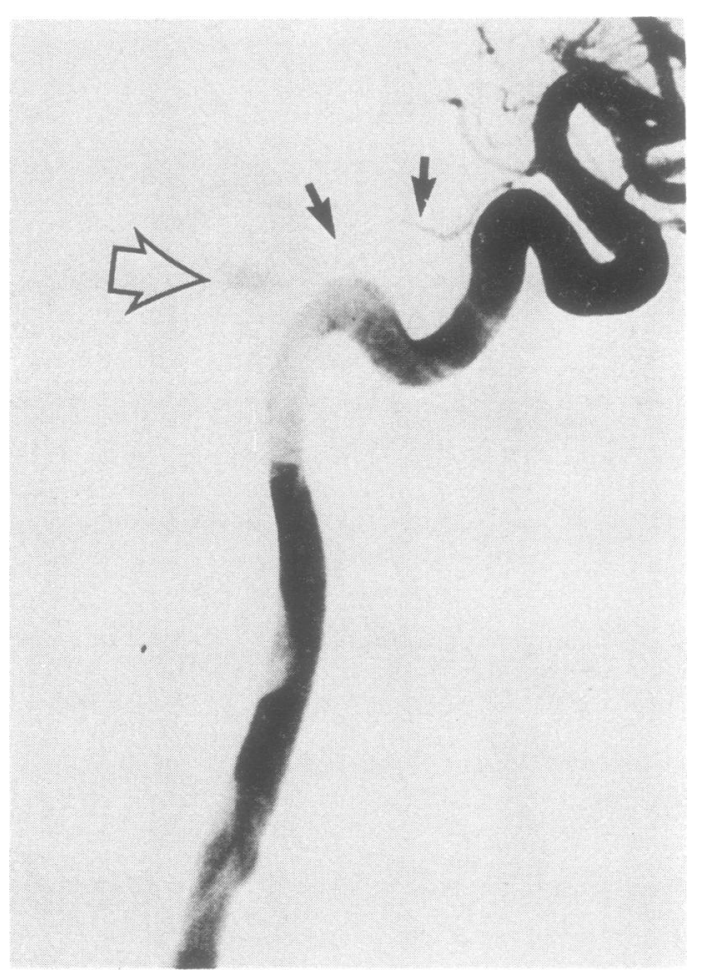

Fig. 10 Case 8 Glomus jugulare tumour-internal carotid angiogram. A small area of pathological circulation is seen in region of middle ear (open arrow), arising from branch of meningohypophyseal artery (arrows). 
processes. It was avulsed piecemeal and the surgeon commented on the fact that the blood loss was significantly less than usual. Recovery was uneventful.

\section{Case 8 Glomus jugulare tumour}

EA, a 46 year old Persian businessman, complained of a pulsatile bruit in his right ear; this had been present for three months, but two years previously he had had one acute attack of vertigo. He had also noticed slight right sided deafness and facial weakness, and mild dysphagia.

These symptoms were confirmed on examination, which also showed a right palatal weakness and wasting of the right side of the tongue. The eardrum was bulging and blue. Although he gave no his'ory of previous operation, there was a large scar overlying the right sternomastoid muscle.

Plain radiographs showed extensive erosion of the right petrous bone. Selective injections of the right vertebral and internal carotid arteries showed very minor contribution to the blood supply of the lesion (Fig. 10). Superselective injection of the branches of the external carotid artery showed the lesion to be fed by two branches of the occipital artery, the ascending pharyngeal artery and the maxillary artery. These four feeding vessels were each selectively embolised (Figs. 11, 12).

The patient was intensely nauseated for two days after embolisation, but made a satisfactory recovery. His symptoms were alleviated and there was some return of bulk to the tongue. Ten months after the first embolisation, he was seen again, complaining that his swallowing had deteriorated.

Plain radiographs indicated that the petrous crosion had increased. Selective vertebral and internal carotid angiograms showed some increase in the supply from these vessels. The occipital

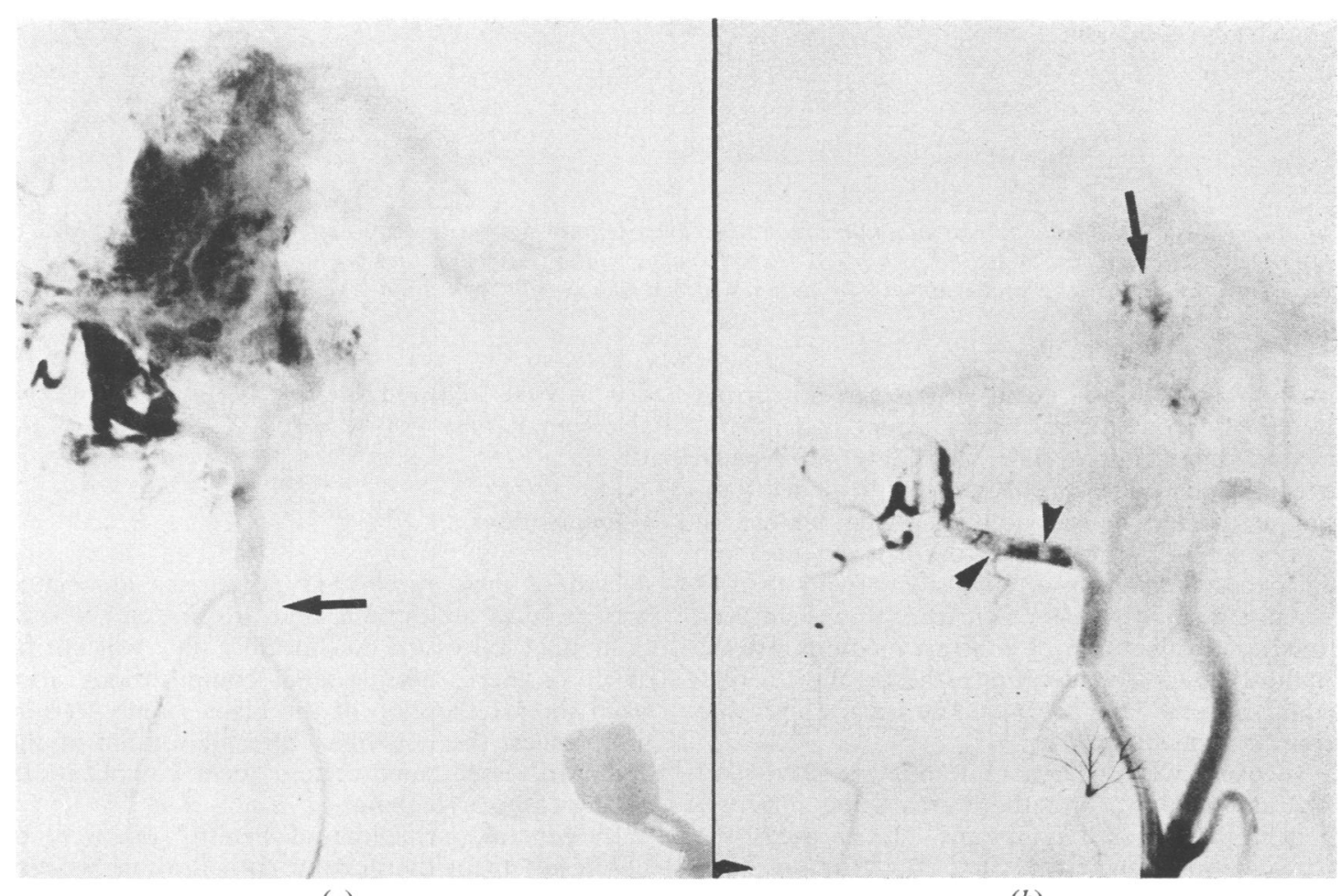

(a)

(b)

Fig. 11 Case 8 Selective injection of occipital artery. (a) The catheter is in main trunk of artery (arrow) from which two branches supply a large tumour mass; (b) after embolisation the larger, posterior branch is occluded and emboli are seen within it (arrowheads). There is still supply to the tumour from the anterior branch; the catheter was advanced into this for further embolisation. Note reflux into other external carotid branches after obliteration of a major portion of tumour. 


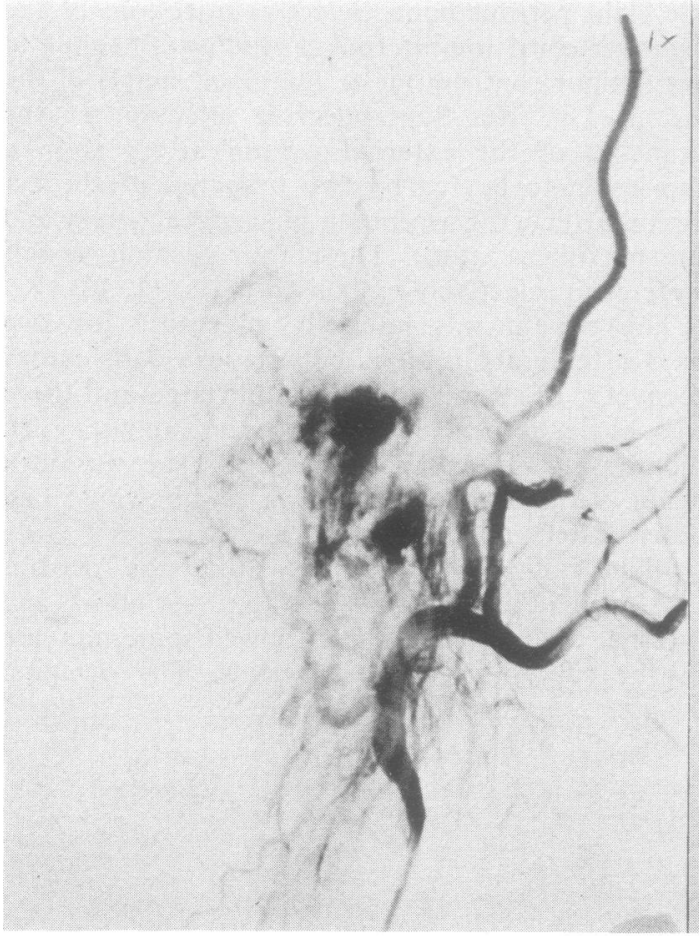

(a)

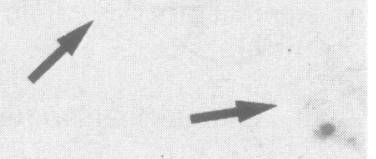

(b)

Fig. 12 Case 8 (a) Injection of maxillary and superficial temporal arteries after embolisation of ascending pharyngeal-further supply to the tumour is seen; (b) after embolisation-a tiny portion of tumour remains. Flush injection shows this and confirms the patency of internal carotid and middle cerebral vessels (arrows).

artery was embolised again, and it was noted that the supply from the maxillary artery had not increased significantly. The ascending pharyngeal artery, which had previously fed only a small portion of the lesion, was now supplying a large tumour mass (Fig. 13a). After the first embolisation and check angiogram of this artery (with $4 \mathrm{ml}$ of contrast medium) it was clear that there had been massive extravasation of contrast medium into the subdural space and even into the cerebral ventricular system (Fig. 13b). The procedure was stopped immediately.

Recovery was uneventful despite the leakage of contrast medium, and there was some improvement in the patient's symptoms. The nature of the extravasation suggested that the tumour had broken from the confines of the petrous bone into the subdural space, and possibly even invaded the subarachnoid space where small veins had ruptured. This, and the obvious extension of the tumour between the two embolisations underline the fact that in such progressive lesions embolisa- tion is best followed by resection when feasible, although it may not be essential (Hekster et al., 1973).

\section{Complications}

All these procedures carry the risks associated with general anaesthesia and arterial catheterisation; since these are non-specific, they will not be dwelt on here. The specific complications arise from the interruption of the blood supply to normal tissues, the possibility of embolisation of the cerebral vessels, and certain local complications of the catheter techniques.

In general, ischaemia of healthy tissues does not result from therapeutic embolisation because of the excellent collateral supply in the regions concerned. However, skin ulceration may occur, particularly around the pinna, and this may be a disadvantage when the procedure is largely cosmetic. Some workers have also described ulceration of the tip of the tongue, a particular hazard 


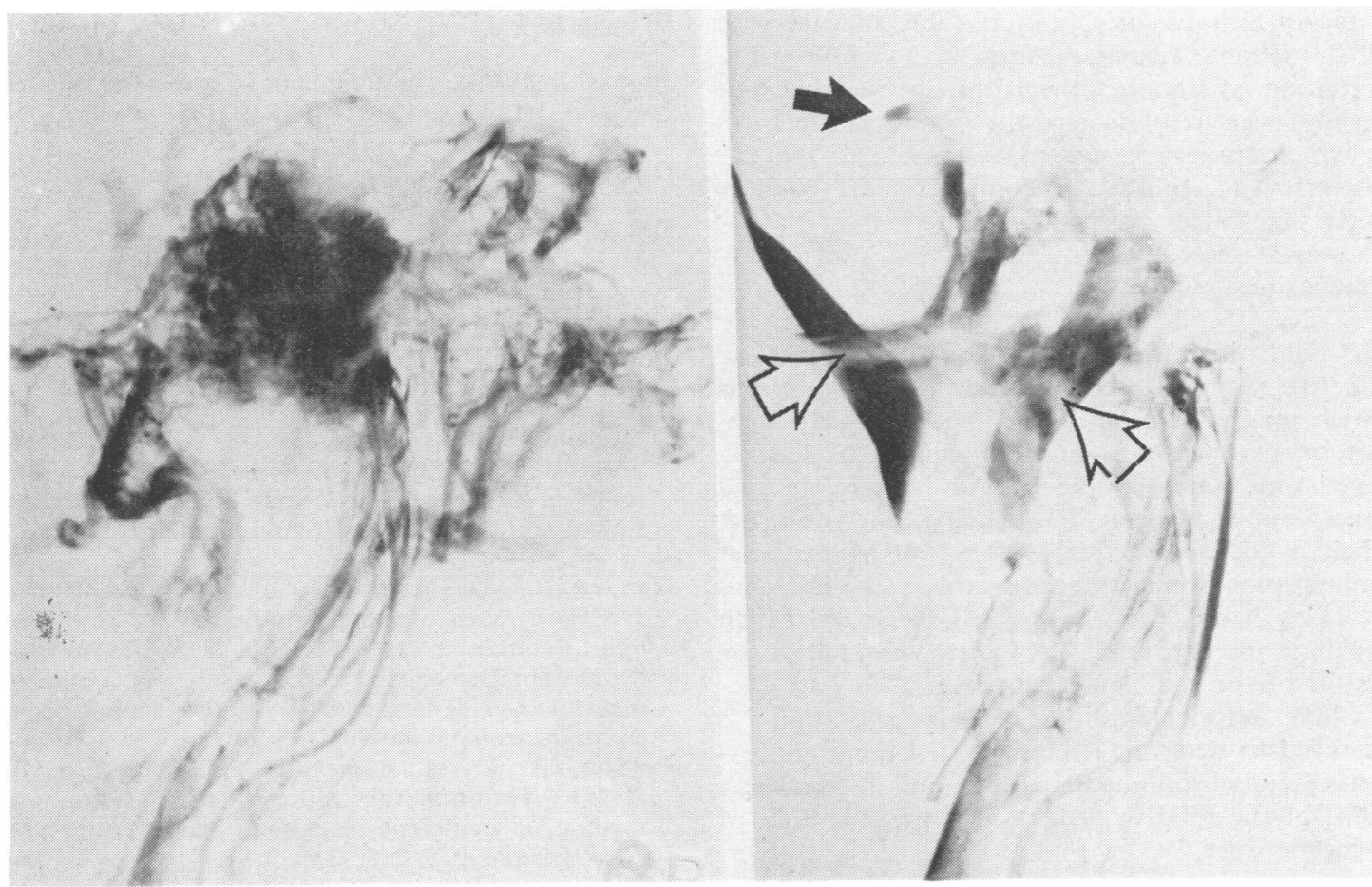

(a)

(b)

Fig. 13 Case 8 Second embolisation. (a) Injection of ascending pharyngeal artery-a considerable regrowth of tumour is seen; (b) after partial embolisation - the tumour vascularity is markedly reduced. but contrast medium is seen outlining the subdural space in front of and behind lower medulla (open arrows), and lying in the dependent portion of the fourth ventricle (arrow).

with extensive bilateral embolisation of the lingual arteries (Djindjian et al., 1973a). In neither of these situations has the ulceration been clinically troublesome. Case 3 of the present paper did show some superficial ulceration of the tonsil after embolisation but this was not significant. In another case, however, preoperative partial embolisation of an enormous facial and pharyngeal angioma was followed 12 hours later by haemorrhage from the larynx; this was thought to be a result of traumatic intubation, but must be regarded as a complication of the procedure. Djindjian et al. (1973b) have also described facial atrophy as a late result of embolisation of facial angiomas or lymphangiomas.

In none of our patients was there any neurological deficit after embolisation of the external carotid artery. Manelfe and his coworkers (Guiraud et al., 1974) described a series of 50 cases in which the internal carotid artery was injected at the end of embolisation in the external carotid territory. Nine cases showed some angiographic abnormality but in only one was this symptomatic. More serious incidents have, however, been described (see Djindjian (1977) for full bibliography). We would emphasise, therefore, that it is vital not to proceed with embolisation unless it appears quite safe; it is also important not to attempt to achieve complete occlusion of all feeding vessels at the time of the procedure, as this clearly sets the stage for reflux into the internal carotid artery.

The local complications include damage to the vessels by intimal tears during attempted superselective catheterisation, or by rupture of small vessels-cf cases 7 and 8 of the present series. Fortunately, neither of these phenomena appears to be clinically significant. After embolisation, some transient pain and swelling of the area usually accompanies a successful result; slight fever is not uncommon. These symptoms are due to thrombosis within the embolised vessels, which usually continues for seven to 14 days after the procedure.

The lesion may become revascularised, par- 
ticularly if it has not been possible to deal with all the feeding vessels. If surgery is to follow embolisation it should, therefore, be carried out within seven to 10 days of the procedure. In cases in which surgery is not contemplated, it may be necessary to repeat the embolisation, possibly more than once.

\section{Conclusions}

It would seem that embolisation represents an effective primary mode of treatment for lesions which may pose problems of bleeding - spontaneous or peroperative-and in which the feeding vessels may not be dealt with effectively and simply by the surgeon. This is particularly relevant when a good cosmetic result is important. That embolisation should precede surgery is illustrated by cases 3 and 4 of the present report, in which previous surgery, while ineffective, prejudiced the results of the radiological procedure.

Close surgical/radiological collaboration is essential to determine the relative timing and extent of embolisation and surgery, and in the assessment of the relative benefits and possible risks of the procedure.

We are grateful to our colleagues at the National Hospital for referring these cases to us and also to Mr R. Pracey, Mr I. W. Broomhead, Mr J. Maynard and Dr R. Heirons for transferring patients to us for the procedure. We thank the Department of Medical Illustration at the Institute of Neurology for preparation of the illustrations and Miss P. M. Hampson for secretarial assistance.

\section{References}

Brooks, B. (1930). The treatment of traumatic arteriovenous fistula. Southern Medical Journal, 23, 100106.

Djindjian, R. (1977). Superselective Arteriography of the External Carotid Artery. Springer: Berlin.

Djindjian, R., Cophignon, J., Théron, J., Merland, J. J., and Houclart, R. (1973a). Embolization by superselective arteriography from the femoral route in neuroradiology. Review of 60 cases. I. Technique, indications, complications. Neuroradiology, 6, 2026.

Djindjian, R., Cophignon, J., Rey, A., Théron, J., Merland, J. J., and Houdart, R. (1973b). Superselective arteriographic embolization by the femoral route in neuroradiology. Study of 50 cases. III. Embolization in craniocerebral pathology. Neuroradiology, 6, 143-152.

Guiraud, B., Manelfe, C., Rascol, A., and Guillaume, J. (1974). Discussion of complications of embolisation. Reunion interrégionale de la Société d'Otoneuro-ophtalmologie de Toulouse: Angiographie supersélective de la carotide externe et embolisation en oto-neuro-ophtalmologie. Toulouse, 26.10.74.

Hekster, R. E. M., Luyendijk, W., Matricali, B. (1973). Transfemoral catheter embolization: a method of treatment of glomus jugulare tumours. Neuroradiology, 5, 208-214.

Ishimori, S., Mattori, M., Shibatta, Y., Sizawa, H., and Fujinaya, R. (1967). Treatment of carotid cavernous fistula by gelfoam embolization. Journal of Neurosurgery, 27, 315-319.

Luessenhop, A. J. (1969). Artificial embolization for cerebral arteriovenous malformations. Progress in Neurological Surgery, 3, 320-362. 